\title{
PROTAC-mediated selective degradation of cytosolic soluble epoxide hydrolase enhances ER-stress reduction
}

Yuxin Wang, ${ }^{1}$ Christophe Morisseau, ${ }^{1}$ Akihiro Takamura, ${ }^{2}$ Debin Wan, ${ }^{1}$ Dongyang Li, ${ }^{1}$ Dennis W. Wolan, ${ }^{2,3 *}$ Bruce D. Hammock, ${ }^{1 *}$ and Seiya Kitamura ${ }^{2,3 *}$

1 Department of Entomology and Nematology, and UC Davis Comprehensive Cancer Center, University of California Davis, One Shields Avenue, Davis, CA 95616, United States

2 Department of Molecular Medicine, The Scripps Research Institute, La Jolla, CA 92037 USA

3 Department of Integrative Structural and Computational Biology, The Scripps Research Institute, La Jolla, CA 92037 USA

* Correspondence: wolan@scripps.edu (D.W.W), bdhammock@ucdavis.edu (B.D.H), skita@scripps.edu (S.K.) 


\section{Highlights}

- $\quad$ First-in-class soluble epoxide hydrolase (sEH) small-molecule degraders.

- Selective degradation of cytosolic but not peroxisomal sEH.

- Significant and stable reduction in SEH protein levels, leading to enhanced cellular efficacy in ER stress reduction relative to the parent inhibitor.

- First example of a PROTAC that induces lysosomal-directed degradation of a soluble target protein.

\section{Summary}

Soluble epoxide hydrolase (sEH) is a bifunctional enzyme responsible for lipid metabolism and is a promising drug target. Here, we report the first-in-class PROTACs small-molecule degraders of sEH. Our optimized PROTAC selectively targets the degradation of cytosolic but not peroxisomal $\mathrm{sEH}$, resulting in exquisite spatiotemporal control. In comparison to the parent sEH inhibitor, sEH-directed PROTAC has higher potency in cellular assays, as measured by significantly reduced ER stress. Surprisingly, PROTAC directs degradation of cytosolic sEH via the lysosome, not through the proteasome, representing the first example of an intracellular lysosomal-dependent PROTAC. The molecules presented here are useful chemical probes to study the biology of sEH with the potential for therapeutic development. Broadly, our results represent a proof-of-concept for the subcellular compartment-selective modulation of a protein class by PROTACs, as well as assisting in the establishment of the cellular mechanism of PROTACs that preferentially direct target proteins for lysosomal degradation. 


\section{Introduction}

Soluble epoxide hydrolase (sEH) is a bifunctional enzyme present in vertebrates and is encoded by the Ephx2 gene (Newman et al., 2003). The N-terminal domain possesses a lipid phosphatase activity, while the C-terminus is responsible for metabolizing epoxy fatty acids to corresponding 1,2-diols (Hashimoto, 2019; Morisseau and Hammock, 2013). Inhibition of sEH hydrolase activity by small molecules (sEHI) is a promising approach for the treatment of multiple diseases including inflammation, pain, cancer, and metabolic disorders (Fishbein et al., 2020; Wagner et al., 2017; Wang et al., 2018). Notwithstanding the extensive biological knowledge and design of small-molecule inhibitors for the sEH domain, the biological importance of the N-terminal phosphatase domain remains to be elucidated.

Mammalian sEH is localized to both the cytosol and peroxisomes (Gill et al., 1994), because sEH encodes for a peroxisomal translocation sequence (PTS) that directs import of sEH into peroxisomes. A previous study indicated sEH contributes to stroke injury only when localized in the cytoplasm, while peroxisomal sEH may be protective, showing the different biological functions of cytosolic and peroxisomal sEH (Nelson et al., 2015). Interestingly, the human sEH is located in both cytosolic and peroxisomal in hepatocytes and renal proximal tubules but exclusively cytosolic in other sEH-containing tissues such as pancreatic islet cells, intestinal epithelium, anterior pituitary cells. These date indicate that sEH subcellular localization is tissue-dependent, and sEH may have tissue- or cell-type specific functions (Enayetallah et al., 2006). Therefore, any molecule that could preferentially label and/or inhibit cytosolic vs. peroxisomal sEH (and vice versa) would be an essential tool to understand the differences in biological importance of sEH between the two cellular locales.

Proteolysis targeting chimera (PROTAC) is a promising technology that promotes the highly selective degradation of target proteins within cells and whole animals (Sakamoto et al., 2001; Sun et al., 2019). PROTACs are heterodimeric molecules that recruit E3 ligase to the proximal location of proteins of interest for ubiquitination and proteasomal-dependent degradation (Sun et al., 2019). In comparison to traditional reversible and covalent small-molecule inhibitors, PROTACs have the additional advantage of labeling and degrading those proteins considered "undruggable", including (but not limited to) transcription factors, transcriptional regulators, and protein-protein interaction partners (Bai et al., 2019; Bond et al., 2020; Garnar-Wortzel et al., 2021; Neklesa et al., 2019). Conversion of established and modest small-molecule protein binders into PROTACs have resulted in improved cellular selectivity and potency. This has significant implications for therapeutics because lower PROTAC concentrations are required for cellular activity relative to the traditional therapeutic counterpart (Salami et al., 2018; Zou et al., 2019). Additionally, PROTACs are "catalytic" and deviate significantly from the mode of actions of traditional competitiveand occupancy-driven inhibitors (Gao et al., 2020). Altogether, small-molecule degraders, including 
PROTACs, are an attractive modality in drug development to overcome the limitations of traditional occupancy-driven ligands.

Here, we report the development of the first-in-class small-molecule degraders of sEH. We demonstrate their ability to reduce sEH levels in a spatio-selective manner and show that the degradation mechanism of sEH PROTAC is lysosome dependent, representing the first PROTAC degrading soluble target protein through the lysosome. Finally, we show enhanced cellular potency of the PROTAC in the cellular ER stress assay in comparison to the parent sEH inhibitor.

\section{Materials and Methods}

\section{Cell Culture and reagents}

Human embryonic kidney 293T cells and human hepatocyte carcinoma $\mathrm{HepG}_{2}$ cells were purchased from American Type Culture Collection (ATCC, Manassas, VA), and the ATG 2A/2B ${ }^{-/}$cell line was a kind gift from Dr. Komatsu Masaaki (Juntendo University, Tokyo, Japan). The cell lines were cultured in highglucose (4.5 g/L) Dulbecco's Modified Eagle's Medium (HyClone, Marlborough, MA). The media was supplemented with $10 \%$ fetal bovine serum (VWR, Radnor, PA), 100 units/mL of penicillin, and 100 $\mathrm{mg} / \mathrm{mL}$ of streptomycin (Thermo Fisher Scientific, Waltham, MA). The cells were cultured at $37{ }^{\circ} \mathrm{C}$ in a $5 \%$ $\mathrm{CO}_{2}$ incubator. Cycloheximide (CHX, \#C1988), MG132 (\#474790), VER155008 (SML0271) were purchased from Sigma-Aldrich (St. Louis, MO), Bortezomib (BTZ, \#NC0175953) was purchased from LC laboratory (Woburn, MA), bafilomycin A1 (BafA1, \#54645S) was purchased from Cell Signaling Technology (Danvers, MA), and the thapsigargin (Tg, \#1138) was purchased from R \& D SYSTEMS INC (Minneapolis, MN).

\section{Protein extraction and immunoblotting}

Human embryonic kidney (HEK) 293T cells and human hepatocyte carcinoma $\mathrm{HepG}_{2}$ cells were treated with SEH PROTAC 1a, DMSO vehicle or negative control 1a' in complete medium. After 24 hours of incubation, the medium was decanted. Then the cells were washed with cold phosphate-buffered saline (PBS) and lysed using RIPA buffer with protease inhibitor cocktail (\#5871S, Cell Signaling, Danvers, MA) and phosphatase inhibitor cocktail (\#78420, Life Technologies, Carlsbad, CA). The cell lysates were then resolved using SDS/PAGE (Bio-Rad, Hercules, CA) and transferred onto a nitrocellulose membrane using the Trans-Blot Turbo Transfer System (\#170-4155, Bio-Rad, Hercules, CA). The membranes were blocked in 5\% fat free milk in PBST for 1 hour at room temperature and probed with the primary antibody. The 
following antibodies were used, including: 1) a previously generated sEH antibody (Imig et al., 2002); 2) phospha-IRE1 $\alpha$ (\#ab48187, Abcam, Waltham, MA); 3) IRE1 $\alpha$ (\#3294, Cell Signaling, Danvers, MA); 4) XBP-1s (\#12782, Cell Signaling, Danvers, MA); and 4) $\beta$-actin (\#A1978, Sigma-Aldrich, St. Louis, MO). The membranes were then probed with goat anti-rabbit and goat anti-mouse secondary antibodies (\#7074, \#7076, Cell Signaling, Danvers, MA), and blots were incubated with Clarity Western ECL Substrate (Cat. \# 170-5061, Bio-Rad, Hercules, CA) and imaged using the ChemiDoc MP (Cat. \# 170-8280, Bio-Rad, Hercules, CA) with ImageLab Version 5 (Bio-Rad, Hercules, CA).

\section{Cellular activity of soluble epoxide hydrolase (sEH)}

The $\mathrm{HepG}_{2}$ cells were treated with different sEH PROTAC compounds and isolated by centrifugation. The cells were suspended in $1 \mathrm{~mL}$ of media. To measure the residual soluble epoxide hydrolase activity $\left[{ }^{3} \mathrm{H}\right]-$ trans-diphenyl-propene oxide ( $t$-DPPO) was used as a substrate (Borhan et al., 1995). One microliter of a $5 \mathrm{mM}$ solution of $t$-DPPO in DMSO was added to $100 \mu \mathrm{L}$ of diluted cell suspension $\left([\mathrm{S}]_{\text {final }}=50 \mu \mathrm{M}\right)$. The mixture was incubated at $37{ }^{\circ} \mathrm{C}$ for $120 \mathrm{~min}$, and the reaction quenched by addition of $60 \mu \mathrm{L}$ of methanol and $200 \mu \mathrm{L}$ of isooctane, which extracts the remaining epoxide from the aqueous phase. Parallel extractions of the stopped reaction with 1-hexanol were performed to assess the possible presence of glutathione transferase activity which could also transform the substrate into a more water soluble glutathione conjugate and give a false positive (Borhan et al., 1995); however, none was observed. The enzyme activity was followed by measuring the quantity of radioactive diol formed in the aqueous phase using a scintillation counter (TriCarb 2810 TR, Perkin Elmer, Shelton, CT). Assays were performed in triplicate. Protein concentration was quantified using the Pierce BCA assay (Pierce, Rockford, IL), using fraction V bovine serum albumin (BSA) as a calibrating standard.

\section{Quantitation of sEH protein level using a PolyHRP ELISA}

The sEH level in $\mathrm{HepG}_{2}$ cells was measured using an ultrasensitive PolyHRP based immunoassay, as previously described (Li et al., 2017). The microplate (No. 442404, Nunc Cat. Thermo Fisher Scientific, Waltham, MA) was coated with anti-human sEH rabbit serum (1:2000 dilution) in $0.05 \mathrm{M} \mathrm{pH} 9.6$ carbonatebicarbonate buffer overnight at $4{ }^{\circ} \mathrm{C}$. Then the plate was blocked with $3 \%(w / v)$ skim milk in PBS for $1 \mathrm{~h}$ at room temperature. Human sEH standards and samples with different dilutions in PBS containing 0.1 $\mathrm{mg} / \mathrm{mL}$ bovine serum albumin (BSA) were then applied in the wells. The biotinylated sEH nanobody (1 $\mu \mathrm{g} / \mathrm{mL}$ ) in PBS was added together to each well immediately to proceed with the immunoreaction for $1 \mathrm{~h}$ at 
room temperature. Then, the SA-PolyHRP in PBS $(25 \mathrm{ng} / \mathrm{mL})$ was applied to continue the reaction for another 30 min after washing. The 3,3',5,5'-tetramethylbenzidine (TMB) substrate was added and incubated for 10-15 $\mathrm{min}$ at room temperature. The reaction was stopped using the color development with $2 \mathrm{M}$ sulfuric acid, the optical density was quantified using a SpectraMax M2 microplate reader (Molecular Devices, Sunnyvale, CA, USA) at $450 \mathrm{~nm}$ within $10 \mathrm{~min}$.

\section{The phosphatase activity of soluble epoxide hydrolase}

The soluble epoxide hydrolase phosphatase activity was determined by quantitative analysis of 1-myristoylglycerol (product) using LC-MS/MS as previously described (Morisseau et al., 2012). Briefly, one $\mu \mathrm{L}$ of a $5 \mathrm{mM}$ solution of 1-myristoyl-glycerol-3-phosphate in water $\left([\mathrm{S}]_{\text {final }}=50 \mu \mathrm{M}\right)$ was added to $100 \mu \mathrm{L}$ of cell extracts. The reaction mixtures were incubated at $37^{\circ} \mathrm{C}$ for 5 to 30 minutes. The reactions were then quenched by adding $100 \mu \mathrm{L}$ of a 50:49:1 mixture of acetonitrile, water and acetic acid containing $200 \mathrm{nM}$ of hexanoyl-ceramide as internal standard. The chromatographic analysis was carried out on a Supelco Discovery "Bio Wide Pore" C-5 RP pre-column (Sigma-Aldrich, St Louis, MO) and the mass spectrometer was operated in scheduled MRM mode with optimized MS transitions as previously described (Morisseau et al., 2012).

\section{Immunofluorescence}

Cells were seeded in the 96-well plate and treated with DMSO, 1a', or PROTAC 1a for $24 \mathrm{~h}$. The cells were fixed with $4 \%$ paraformaldehyde for 20 min and cells were permeabilized using $0.02 \%$ Triton X-100 in PBS. After this, the cells were blocked with 5\% goat serum, and 1\% bovine serum albumin in PBS for 1 hour at room temperature. Then, the cells were incubated with specific primary antibodies at $4{ }^{\circ} \mathrm{C}$ overnight and were subsequently incubated with the corresponding secondary antibodies for $1 \mathrm{~h}$ at room temperature. The nuclei were counterstained for 10 min with DAPI. Images were acquired at 20x magnification using the ImageXpress Micro XL high-content imaging system (Molecular Devices, Sunnyvale, CA), and automated image analysis was performed using Custom Module Editor, MetaXpress software (Molecular Devices, version 6.2, RRID:SCR_016654). The following primary antibodies were used in this experiment: mouse monoclonal anti-sEH (sc-166961, Santa Cruz Biotechnology, Dallas, TX), LAMP2 rabbit mAb (\#49067, Cell signaling, Danvers, MA), $\beta$-tubulin antibody (\#2146, Cell signaling, Danvers, MA), Catalase rabbit mAb (\#12980, Cell signaling, Danvers, MA). The following secondary antibodies were purchased 
from Invitrogen: Alexa-Fluor 647 (1:500 dilution, Thermo Fisher, Waltham, MA), goat anti-rabbit conjugated to Alexa-Fluor 488 (1:500 dilution, Thermo Fisher, Waltham, MA).

\section{Data Analysis}

Descriptive statistics were obtained and include mean and standard error of mean for continuous variables and count and proportion for categorical variables. Pairwise comparisons in changes in protein levels and ER stress were performed and include a Student's t-test for continuous variables and Mann-Whitney test for categorical variables. Comparisons across all groups were made via an ANOVA. The details of statistical analyses are given in the figure legend and supporting information.

\section{Results}

\section{Development of SEH PROTACs}

Two series of sEH PROTAC compounds were synthesized based on sEH inhibitors trans-4-[4-(3trifluoromethoxyphenyl-1-ureido)-cyclohexyloxy]-benzoic acid (t-TUCB 1, series 1) (Hwang et al., 2007) and 12-(3-adamantan-1-yl-ureido) dodecanoic acid (AUDA 2, series 2) (Morisseau et al., 1999). Recruiters of E3 ligases (Von Hippel-Lindau (VHL) or cereblon (CRBN)) were connected to sEH binders with various linkers (Table 1). Negative control compounds (1a' and 2a', Table 1) were designed based on compounds 1a and 2a; while they bind to $\mathrm{sEH}$, they do not possess the ability to activate degradation of the target protein.

The inhibitory potency against the human and mouse sEH was determined (Table 1 and Table S1). All the molecules showed low to sub nanomolar potency against sEH hydrolase activity, suggesting that the addition of the linker and PROTAC recruiter does not significantly affect compound binding. This is consistent with previous studies showing that a central urea or amide with two lipophilic substitutions is the minimal structural requirement for the sEH inhibition (Imig and Hammock, 2009; Kitamura et al., 2017; Kitamura et al., 2015; Morisseau et al., 1999). Because these molecules are based on the inhibitors of sEH hydrolase, none of them showed inhibition against sEH phosphatase activity as expected (Table $\mathbf{1}$ and Table S1).

Encouraged by these biochemical data, the sEH protein degradation potency was examined next in a cell-based assay using four complementary methods of sEH detection. The human hepatocyte carcinoma cell line $\mathrm{HepG}_{2}$ was treated with $1 \mu \mathrm{M}$ sEH PROTAC compounds for $24 \mathrm{~h}$. The degradation of sEH was first monitored using immunoblotting. PROTAC compounds 1a, 1c, 1e, 2a, 2c, 2d, 2e showed degradation of sEH in this assay (Figure 1A). There is a correlation of the degradation potency of the molecules with 
the same linker/E3 recruiter between series 1 and series 2. For example, a thalidomide recruiter with PEG4 linker (1a, 2a) seems to show the highest degradation in each series. These six compounds (1a, 1c, 1e, 2a, 2c, 2d, 2e) were chosen for further analysis. Enzymatic activities were next used to monitor the reduction of the levels of functional sEH (Figures 1B, 1C, and Table S2). The parent sEH inhibitors 1 and $\mathbf{2}$ resulted in only slight inhibition of the hydrolase activity. These compounds are reversible inhibitors, thus were probably eliminated during the washing step of the cells with fresh media. Thus, the low inhibition observed is most likely resulting from the residual inhibitor which remained in the buffer, on the plastic wells, or on the enzyme active site. Consistent with the immunoblotting data, compounds 1a and 2a yielded the greatest loss of both hydrolase and phosphatase activity. Finally, we used enzyme-linked immunosorbent assay (ELISA), which can quantitatively determine the absolute concentration of sEH protein in a highthroughput manner (Li et al., 2017). Consistent with other methods, compounds 1a and 2a showed the lowest level of sEH protein, suggesting the highest degradation potency (Figure 1D and Table S2). Overall, compounds 1a showed high degradation efficacy, thus this molecule was selected for further study. To determine the degradation potency, we performed a dose-response $(5-1,000 \mathrm{nM})$ of sEH PROTAC 1a in $\mathrm{HepG}_{2}$ cells (Figure 1E). In addition, the degradation efficacy of $1 \mathbf{a}$ at $250 \mathrm{nM}$ was determined in both $\mathrm{HepG}_{2}$ and $293 \mathrm{~T}$ cells, showing that the compound is effective in both cell lines (Figure 1F). A significantly lower amount of sEH was observed at concentrations as low as $25 \mathrm{nM}$ (Figure 1E). As the dose was increased, the sEH protein band became lighter to reach a maximal degradation at $250 \mathrm{nM}$, then slightly less degradation at $500 \mathrm{nM}$ and $1 \mu \mathrm{M}$. The U-shaped concentration-response curve, also known as the hook effect, is a well-known phenomenon observed previously with other PROTACs (Pettersson and Crews, 2019). Interestingly, the treatment failed to reach full elimination of the sEH protein band, even at the maximum effective dose.

\section{PROTAC 1a selectively degrades cytosolic $\mathrm{SEH}$ but not peroxisome $\mathrm{SEH}$}

To explain this apparent lack of total efficacy and given that sEH has a dual subcellular localization (Enayetallah et al., 2006; Moody et al., 1985), we hypothesized that 1a induced the degradation of only cytosolic sEH but not the peroxisomal sEH. To test this, the cell lysates were separated into two fractions, a cytosol-containing fraction (S10) and peroxisome containing fraction (P10). The immunoblot analysis of both fractions showed that 1a degraded cytosolic sEH while the peroxisomal sEH is not degraded (Figure 2A). The hydrolase activity assay and ELISA results confirmed that compound 1a selectively degraded the cytosolic sEH (Figure 2B and 2C). Consistent with this, immunofluorescence imaging showed that compound 1a decreased the colocalization between sEH and the cytosol marker $\beta$-tubulin but did not affect the sEH colocalization with the peroxisome marker catalase, further supporting the spatial selectivity of the sEH-PROTAC. (Figure 2D and Figure S1). 


\section{Probing the kinetics of degradation and production of SEH using PROTACs}

Next, the kinetics of sEH degradation induced by 1a was measured to probe the cellular turnover of sEH. Surprisingly, compound 1a required 24 h to yield the highest level of degradation (Figure 3A and Figure S2), unlike previously reported PROTACs that show degradation within $30 \mathrm{~min}$ (Liu et al., 2020). For comparison, the endogenous degradation kinetics of sEH was analyzed using cycloheximide (CHX), the protein synthesis inhibitor (Schneider-Poetsch et al., 2010). The results showed that it requires $48 \mathrm{~h}$ to degrade half of the sEH in the cellular system (Figure 3B), indicating that, even slow, sEH-PROTAC compound 1a accelerates the degradation of $\mathrm{sEH}$. In addition, there was no significant difference between CHX treatment with/without the proteasome inhibitor bortezomib (BTZ), suggesting that the endogenous $\mathrm{sEH}$ is not degraded through proteasome (Figure 3C).

We next investigated the kinetics of $\mathrm{sEH}$ production. $\mathrm{HepG}_{2}$ cells were treated with 1a, then the compound was removed by changing the medium. Cells were incubated in media without PROTAC molecules and collected at different time points. The sEH levels were monitored using the ELISA. The results showed that treatment with 1a reduced the level of sEH protein for 72 hours (Figure 3D). Altogether, these data show the slow turnover of $\mathrm{sEH}$ in the cells and give insights into the endogenous degradation mechanisms and kinetics of sEH.

\section{Compound 1a degrades sEH through lysosome dependent pathway}

PROTACs are believed to trigger the proteasome-dependent degradation of target proteins (Lai and Crews, 2017), thus proteasome inhibitors should be able to rescue the target protein from the degradation effects. To test the mechanism of protein degradation, $\mathrm{HepG}_{2}$ cells were treated with proteasome inhibitors BTZ or MG132 with PROTAC 1a. Interestingly, both BTZ and MG132 failed to block the degradation induced by PROTAC 1a (Figures 4A and 4B), suggesting that 1a induced degradation for sEH is not through the proteasome. Rather, BTZ promoted endogenous sEH degradation (Figure 4A). Several studies have reported compensation mechanism between proteasome and autophagy to maintain cellular homeostasis ( $\mathrm{Ji}$ and Kwon, 2017). Taken together with the slow kinetics, we speculated that sEH is degraded through the lysosomal pathway. To test this possibility, the cells were co-treated with the autophagolysosome inhibitor bafilomycin A1 (BafA1). BafA1 blocked the degradation induced by 1a in both $\mathrm{HepG}_{2}$ and 293T cells (Figure 4C and Figure S3). Furthermore, immuno-histochemical imaging clearly showed that 1a treatment promoted SEH colocalization with LAMP2, a lysosome marker, and cotreatment with BafA1 reversed this trend (Figure 4D), supporting that the $\mathrm{sEH}$ degradation is lysosome dependent.

Lysosome-dependent protein degradation pathways are divided into three major classes; macroautophagy, endocytosis/microautophagy, and chaperon-mediated autophagy (Huber and Teis, 2016). 
We tested these previously described lysosomal pathways to determine the exact degradation mechanisms, including using ATG2A/2B ${ }^{-1}$ 293T cell or knockdown ATG5 in 293T cell (macroautophagy), knockdown of the tumor susceptibility gene 101 (tsg101) (endocytosis/microautophagy), and Hsp70 family inhibitor VER155008 (microautophagy and chaperon mediated autophagy) (Figure S4). All these treatments failed to rescue the $\mathrm{sEH}$ degradation induced by 1a, indicating that its degradation is through a previously uncharacterized lysosomal degradation pathway, or, alternatively, there is an unknown compensation mechanism for sEH degradation. Determining the exact lysosomal dependent degradation pathway is the subject of further study.

\section{Compound 1a rescued cell viability through inhibition of IRE1 $\alpha$-XBP1 signaling pathway.}

Previous studies demonstrated that sEH is a physiological modulator of ER stress signaling (Bettaieb et al., 2013; Inceoglu et al., 2017; Wang et al., 2021). Thus, the efficacy of the sEH-PROTAC compounds was tested in the cellular thapsigargin (Tg)-induced ER stress assay focusing on the IRE1 $\alpha$-XBP1s pathway. To maximize the efficacy, we first pretreated the cells with PROTAC 1a or 1a' for 24 hours, then after removing the compounds, the cells were treated with Tg for 24 hours to induce ER stress. PROTAC 1a decreased the phosphorylated IRE1 $\alpha$ and the downstream splicing XBP1 in both $\mathrm{HepG}_{2}$ and 293T cells (Figure 5A). Consistent with immunoblot results, 1a also rescued the cell death induced by ER stress (Figure 5B). In addition, compound 1a has better efficacy on rescuing cell death when treated with $\mathrm{Tg}$ together for 24 hours. While for the longer time treatment, there is no significant difference between $\mathbf{1}$ and 1a for the rescuing effect (Figure S5), further supporting PROTAC 1a rescued cell viability induced by Tg.

\section{Discussion}

We here report the development of the first-in-class small molecule degraders of sEH and their application to study the biology of sEH. For the development of the PROTAC molecules, we employed two scaffolds of sEH inhibitors, AUDA and $t$-TUCB (Table 1). AUDA is one of the first generation sEH inhibitors with high potency against most mammalian sEH homologs while $t$-TUCB is a newer inhibitor with improved physicochemical properties and metabolic stability (Hwang et al., 2007; Morisseau et al., 2002). In both cases, previous SAR and x-ray complex structures showed that carboxylic acid is not required for potency. Therefore, we connected the carboxylic acid to the recruiters of widely used E3 ligases (Von Hippel-Lindau (VHL) or cereblon (CRBN)) with various linker lengths and types (alkyl chain or PEG) to optimize the degradation potency. 
For the prioritization of the synthesized molecules, four complementary assays were used to detect the sEH levels. Interestingly, while globally similar, there are a few discrepancies among the data from these four methods. For example, compound 1e showed high degradation effects as monitored by the immunoblotting and sEH hydrolase activity, while only the limited effects were observed in the phosphatase activity and ELISA. A possible explanation for these apparently contradictory results includes that this molecule induces partial degradation of $\mathrm{sEH}$, specifically degrading hydrolase domain and not phosphatase domain. Immunoblotting differentiates the partial degradation vs full degradation of sEH while ELISA may not be able to, assuming that the nanobody recognizes the epitope on the phosphatase domain. Determining the exact mechanism of this interesting observation is the subject of further study. Nevertheless, in all assays, compound 1a showed high degradation efficacy. For future screening and optimization of sEH PROTACs, the ELISA assay may be the method of choice given the throughput, robustness, and quantitative aspects.

The sEH-PROTAC decreased the enzyme activity of both hydrolase and phosphatase activities (Figures 1B and 1C). The phosphatase domain has been shown to metabolize the lysophosphatidic acids (Morisseau et al., 2012), regulate the subcellular localization of sEH (Kramer and Proschak, 2017), and negatively regulate simvastatin-activated eNOS by impeding the Akt-AMPK-eNOS signaling cascade (Hou et al., 2015). The development of potent small-molecule phosphatase inhibitors has been challenging. As an alternative approach, the molecules developed here could be useful chemical probes to study the role of sEH phosphatase activity in the cytosol vs peroxisome.

One of our central findings is that SEH PROTAC selectively instigates the degradation of cytosolic sEH while peroxisomal sEH is resistant to degradation. It is reported that peroxisomal translocation of soluble epoxide hydrolase protects against ischemic stroke injury (Nelson et al., 2015), and sEH dimerization status is a key regulator of its peroxisomal localization (Nelson et al., 2016). The sEH PROTAC compounds will be useful chemical probes to understand the functional difference of peroxisomal vs cytosolic sEH. Furthermore, our study showcases the proof of concept of selective modulation of the protein function in a subcellular-component-specific manner using PROTACs. Cytosol selective degradation is likely due to the inaccessibility of the E3-ligase complex and/or proteasome/lysosome to the inside of the peroxisome. So far, PROTACs have been developed to target cytosolic proteins, nuclear proteins, endosomal membrane proteins, single- and multi-pass transmembrane proteins (Bai et al., 2019; Bond et al., 2020; Neklesa et al., 2019), while PROTACs have not been reported to target mitochondrial proteins, ER-localized proteins, and Golgi-localized proteins. All these proteins that are not degradable by PROTACs are within a membranesurrounded subcellular compartment. Developing small molecule degraders of these proteins is a challenge for future research. 
The sEH PROTAC compounds are thalidomide- or VHL ligand-based PRTOACs that induce protein degradation through the ubiquitin-proteasome system (UPS) (Bondeson et al., 2018; Smith et al., 2019). However, interestingly in our case, co-treatment of proteasome inhibitors with compound 1a did not rescue sEH. Instead, the proteasome inhibitor treatment promoted sEH degradation (Figures $4 \mathbf{A}$ and 4B). Surprisingly, lysosomal inhibitor BafA rescued sEH, suggesting that sEH is degraded through the lysosomal pathway (Figures 4C and S3). Further research is needed to determine the exact mechanisms of sEH degradation induced by compound $\mathbf{1 a}$.

The sEH PROTAC compound described herein is the first PROTAC that induces the degradation of a soluble target protein through the lysosome. A recent study showed that PROTACs targeting a membrane protein EGFR also degrade through a lysosome-dependent manner (Qu et al., 2021), which indicates some of the PROTACs may induce proteasome independent and lysosome dependent degradation. Based on this, it is strongly recommended to test the degradation mechanism of PROTACs using proteasome inhibitors. Endogenous ubiquitination of proteins sometimes leads to lysosomal degradation, while the exact determinant of the specific degradation mechanism is still unclear. For example, it is known that some longlived proteins are endogenously degraded through the lysosome. Consistent with previous proteomic analysis, the cellular turnover of sEH appeared to be slow (Figure 3) (Mathieson et al., 2018). At this point, it is unclear whether the lysosome-dependent degradation is the only degradation mechanism for sEH, or the degradation mechanism is PROTAC structure-dependent. Our findings, together with the study on PROTAC-induced lysosome-dependent EGFR degradation (Qu et al., 2021), suggest that the PROTACs can also induce lysosome-dependent degradation of target proteins and caution the current assumption of the mechanism of action of PROTACs.

Finally, we demonstrated that the SEH PROTAC compound has enhanced potency compared to the parent inhibitor using a cellular ER stress assay. This is likely due to the PROTACs-induced sustained degradation of sEH during ER stress. This could be advantageous for clinical applications through reducing undesirable side effect with prolonged efficacy. Determining the in vivo efficacy of the developed PROTACs is the subject of further study.

In summary, the sEH-PROTAC compounds reported herein selectively degrades cytosolic sEH and not peroxisomal sEH, providing a useful tool to study the function of $\mathrm{sEH}$ in a spatiotemporal manner. Furthermore, the data indicate that our PROTAC compound induces lysosome-dependent, proteasomeindependent degradation of the targeted protein, shedding light on the lysosomal-dependent degradation pathway induced by PROTACs and questioning the current mechanistic assumption of PROTACs. 
Acknowledgments: We thank Dr. Ana Cristina G Grodzki for assistance with immunofluorescence, and grant P50HD103526 from IDDRC- MIND Institute, UC Davis for the use of the imaging facility from the Biological and Molecular Analysis Core (BMAC). We would like to thank Dr. Ian Wilson and Dr. K. Barry Sharpless for support and encouragement on this project and access to the instrument. We also thank Dr. Komatsu Masaaki for kindly providing the ATG $2 \mathrm{~A} / 2 \mathrm{~B}^{-{ }^{-}}$cells, Dr. Sung Hee Hwang for kindly providing the sEH inhibitors $t$-TUCB and AUDA, and Dr. Natalia Orendain for her help on statistical data analysis. We would also like to thank Dr. Takanori Otomo for helpful discussion and help on obtaining cells and project management. This study was supported, in part, by a grant from the National Institute of Environmental Health Sciences (NIEHS) Grant R35ES030443 (B.D.H.), NIEHS Superfund Research Program P42 ES004699 (B.D.H.), and National Institute of General Medical Sciences of the National Institutes of Health under Award Number R35GM136286 (D.W.W.), K99GM138758 (S.K.).

Author contributions: Conceptualization: Y.W., C.M., S.K.; Synthesis: S.K., A.T.; Data acquisition and analysis: Y.W., C.M., D.W., D.L., S.K.; Visualization: Y.W. and S.K.; Project administration: C.M., S. K.; Writing — original draft preparation: Y.W., S.K. Supervision: D.W.W., B.D.H.. All authors have read, reviewed, edited, and agreed to the published version of the manuscript.

Declaration of interests: C.M. and B.D.H. are inventors on patents related to the use of sEH inhibitors owned by the University of California.

\section{References}

Bai, L., Zhou, H., Xu, R., Zhao, Y., Chinnaswamy, K., McEachern, D., Chen, J., Yang, C.Y., Liu, Z., Wang, M., et al. (2019). A Potent and Selective Small-Molecule Degrader of STAT3 Achieves Complete Tumor Regression In Vivo. Cancer Cell 36, 498-511 e417. 10.1016/j.ccell.2019.10.002.

Bettaieb, A., Nagata, N., AbouBechara, D., Chahed, S., Morisseau, C., Hammock, B.D., and Haj, F.G. (2013). Soluble epoxide hydrolase deficiency or inhibition attenuates diet-induced endoplasmic reticulum stress in liver and adipose tissue. J Biol Chem 288, 14189-14199. 10.1074/jbc.M113.458414.

Bond, M.J., Chu, L., Nalawansha, D.A., Li, K., and Crews, C.M. (2020). Targeted Degradation of Oncogenic KRAS(G12C) by VHL-Recruiting PROTACs. ACS Cent Sci 6, 1367-1375.

10.1021/acscentsci.0c00411.

Bondeson, D.P., Smith, B.E., Burslem, G.M., Buhimschi, A.D., Hines, J., Jaime-Figueroa, S., Wang, J., Hamman, B.D., Ishchenko, A., and Crews, C.M. (2018). Lessons in PROTAC Design from Selective Degradation with a Promiscuous Warhead. Cell Chem Biol 25, 78-87 e75.

10.1016/j.chembiol.2017.09.010.

Borhan, B., Mebrahtu, T., Nazarian, S., Kurth, M.J., and Hammock, B.D. (1995). Improved radiolabeled substrates for soluble epoxide hydrolase. Anal Biochem 231, 188-200. 10.1006/abio.1995.1520. 
Enayetallah, A.E., French, R.A., Barber, M., and Grant, D.F. (2006). Cell-specific subcellular localization of soluble epoxide hydrolase in human tissues. J Histochem Cytochem 54, 329-335.

10.1369/jhc.5A6808.2005.

Fishbein, A., Wang, W., Yang, H., Yang, J., Hallisey, V.M., Deng, J., Verheul, S.M.L., Hwang, S.H., Gartung, A., Wang, Y., et al. (2020). Resolution of eicosanoid/cytokine storm prevents carcinogen and inflammation-initiated hepatocellular cancer progression. Proc Natl Acad Sci U S A 117, 21576-21587. 10.1073/pnas.2007412117.

Gao, H., Sun, X., and Rao, Y. (2020). PROTAC Technology: Opportunities and Challenges. ACS Med Chem Lett 11, 237-240. 10.1021/acsmedchemlett.9b00597.

Garnar-Wortzel, L., Bishop, T.R., Kitamura, S., Milosevich, N., Asiaban, J.N., Zhang, X., Zheng, Q., Chen, E., Ramos, A.R., Ackerman, C.J., et al. (2021). Chemical Inhibition of ENL/AF9 YEATS Domains in Acute Leukemia. ACS Cent Sci 7, 815-830. 10.1021/acscentsci.0c01550.

Gill, S., Grant, D.F., Beetham, J.K., Chang, C., and Hammock, B.D. (1994). Peroxisomal proliferation and subcellular localization of soluble epoxide hydrolase: Role of peroxisomal targeting sequences. Peroxisome Proliferators: Unique Inducers of Drug-Metabolizing Enzymes, 113-121.

Hashimoto, K. (2019). Role of Soluble Epoxide Hydrolase in Metabolism of PUFAs in Psychiatric and Neurological Disorders. Front Pharmacol 10, 36. 10.3389/fphar.2019.00036.

Hou, H.H., Liao, Y.J., Hsiao, S.H., Shyue, S.K., and Lee, T.S. (2015). Role of phosphatase activity of soluble epoxide hydrolase in regulating simvastatin-activated endothelial nitric oxide synthase. Sci Rep 5 , 13524. 10.1038/srep13524.

Huber, L.A., and Teis, D. (2016). Lysosomal signaling in control of degradation pathways. Curr Opin Cell Biol 39, 8-14. 10.1016/j.ceb.2016.01.006.

Hwang, S.H., Tsai, H.J., Liu, J.Y., Morisseau, C., and Hammock, B.D. (2007). Orally bioavailable potent soluble epoxide hydrolase inhibitors. J Med Chem 50, 3825-3840. 10.1021/jm070270t.

Imig, J.D., and Hammock, B.D. (2009). Soluble epoxide hydrolase as a therapeutic target for cardiovascular diseases. Nat Rev Drug Discov 8, 794-805. 10.1038/nrd2875.

Imig, J.D., Zhao, X., Capdevila, J.H., Morisseau, C., and Hammock, B.D. (2002). Soluble epoxide hydrolase inhibition lowers arterial blood pressure in angiotensin II hypertension. Hypertension 39, 690694. 10.1161/hy0202.103788.

Inceoglu, B., Bettaieb, A., Haj, F.G., Gomes, A.V., and Hammock, B.D. (2017). Modulation of mitochondrial dysfunction and endoplasmic reticulum stress are key mechanisms for the wide-ranging actions of epoxy fatty acids and soluble epoxide hydrolase inhibitors. Prostaglandins Other Lipid Mediat 133, 68-78. 10.1016/j.prostaglandins.2017.08.003.

Ji, C.H., and Kwon, Y.T. (2017). Crosstalk and Interplay between the Ubiquitin-Proteasome System and Autophagy. Mol Cells 40, 441-449. 10.14348/molcells.2017.0115.

Kitamura, S., Morisseau, C., Harris, T.R., Inceoglu, B., and Hammock, B.D. (2017). Occurrence of ureabased soluble epoxide hydrolase inhibitors from the plants in the order Brassicales. PLoS One 12, e0176571. 10.1371/journal.pone.0176571.

Kitamura, S., Morisseau, C., Inceoglu, B., Kamita, S.G., De Nicola, G.R., Nyegue, M., and Hammock, B.D. (2015). Potent natural soluble epoxide hydrolase inhibitors from Pentadiplandra brazzeana baillon: synthesis, quantification, and measurement of biological activities in vitro and in vivo. PLoS One 10, e0117438. 10.1371/journal.pone.0117438.

Kramer, J., and Proschak, E. (2017). Phosphatase activity of soluble epoxide hydrolase. Prostaglandins Other Lipid Mediat 133, 88-92. 10.1016/j.prostaglandins.2017.07.002.

Lai, A.C., and Crews, C.M. (2017). Induced protein degradation: an emerging drug discovery paradigm.

Nat Rev Drug Discov 16, 101-114. 10.1038/nrd.2016.211.

Li, D., Cui, Y., Morisseau, C., Gee, S.J., Bever, C.S., Liu, X., Wu, J., Hammock, B.D., and Ying, Y. (2017). Nanobody Based Immunoassay for Human Soluble Epoxide Hydrolase Detection Using Polymeric Horseradish Peroxidase (PolyHRP) for Signal Enhancement: The Rediscovery of PolyHRP? Anal Chem 89, 6248-6256. 10.1021/acs.analchem.7b01247. 
Liu, J., Chen, H., Ma, L., He, Z., Wang, D., Liu, Y., Lin, Q., Zhang, T., Gray, N., Kaniskan, H.U., et al. (2020). Light-induced control of protein destruction by opto-PROTAC. Sci Adv 6, eaay5154.

10.1126/sciadv.aay5154.

Mathieson, T., Franken, H., Kosinski, J., Kurzawa, N., Zinn, N., Sweetman, G., Poeckel, D., Ratnu, V.S., Schramm, M., Becher, I., et al. (2018). Systematic analysis of protein turnover in primary cells. Nat Commun 9, 689. 10.1038/s41467-018-03106-1.

Moody, D.E., Loury, D.N., and Hammock, B.D. (1985). Epoxide metabolism in the liver of mice treated with clofibrate (ethyl-alpha-(p-chlorophenoxyisobutyrate)), a peroxisome proliferator. Toxicol Appl Pharmacol 78, 351-362. 10.1016/0041-008x(85)90240-6.

Morisseau, C., Goodrow, M.H., Dowdy, D., Zheng, J., Greene, J.F., Sanborn, J.R., and Hammock, B.D. (1999). Potent urea and carbamate inhibitors of soluble epoxide hydrolases. Proc Natl Acad Sci U S A 96, 8849-8854. 10.1073/pnas.96.16.8849.

Morisseau, C., Goodrow, M.H., Newman, J.W., Wheelock, C.E., Dowdy, D.L., and Hammock, B.D. (2002). Structural refinement of inhibitors of urea-based soluble epoxide hydrolases. Biochem Pharmacol 63, 1599-1608. 10.1016/s0006-2952(02)00952-8.

Morisseau, C., and Hammock, B.D. (2013). Impact of soluble epoxide hydrolase and epoxyeicosanoids on human health. Annu Rev Pharmacol Toxicol 53, 37-58. 10.1146/annurev-pharmtox-011112-140244. Morisseau, C., Schebb, N.H., Dong, H., Ulu, A., Aronov, P.A., and Hammock, B.D. (2012). Role of soluble epoxide hydrolase phosphatase activity in the metabolism of lysophosphatidic acids. Biochem Biophys Res Commun 419, 796-800. 10.1016/j.bbrc.2012.02.108.

Neklesa, T., Snyder, L.B., Willard, R.R., Vitale, N., Pizzano, J., Gordon, D.A., Bookbinder, M., Macaluso, J., Dong, H., Ferraro, C., et al. (2019). ARV-110: An oral androgen receptor PROTAC degrader for prostate cancer. Journal of Clinical Oncology 37, 259-259.

10.1200/JCO.2019.37.7_suppl.259.

Nelson, J.W., Das, A.J., Barnes, A.P., and Alkayed, N.J. (2016). Disrupting Dimerization Translocates Soluble Epoxide Hydrolase to Peroxisomes. PLoS One 11, e0152742. 10.1371/journal.pone.0152742. Nelson, J.W., Zhang, W., Alkayed, N.J., and Koerner, I.P. (2015). Peroxisomal translocation of soluble epoxide hydrolase protects against ischemic stroke injury. J Cereb Blood Flow Metab 35, 1416-1420. $10.1038 / \mathrm{jcbfm} .2015 .159$.

Newman, J.W., Morisseau, C., Harris, T.R., and Hammock, B.D. (2003). The soluble epoxide hydrolase encoded by EPXH2 is a bifunctional enzyme with novel lipid phosphate phosphatase activity. Proc Natl Acad Sci U S A 100, 1558-1563. 10.1073/pnas.0437724100.

Pettersson, M., and Crews, C.M. (2019). PROteolysis TArgeting Chimeras (PROTACs) - Past, present and future. Drug Discov Today Technol 31, 15-27. 10.1016/j.ddtec.2019.01.002.

Qu, X., Liu, H., Song, X., Sun, N., Zhong, H., Qiu, X., Yang, X., and Jiang, B. (2021). Effective degradation of EGFR(L858R+T790M) mutant proteins by CRBN-based PROTACs through both proteosome and autophagy/lysosome degradation systems. Eur J Med Chem 218, 113328. 10.1016/j.ejmech.2021.113328.

Sakamoto, K.M., Kim, K.B., Kumagai, A., Mercurio, F., Crews, C.M., and Deshaies, R.J. (2001). Protacs: chimeric molecules that target proteins to the Skp1-Cullin-F box complex for ubiquitination and degradation. Proc Natl Acad Sci U S A 98, 8554-8559. 10.1073/pnas.141230798.

Salami, J., Alabi, S., Willard, R.R., Vitale, N.J., Wang, J., Dong, H., Jin, M., McDonnell, D.P., Crew, A.P., Neklesa, T.K., and Crews, C.M. (2018). Androgen receptor degradation by the proteolysis-targeting chimera ARCC-4 outperforms enzalutamide in cellular models of prostate cancer drug resistance.

Commun Biol 1, 100. 10.1038/s42003-018-0105-8.

Schneider-Poetsch, T., Ju, J., Eyler, D.E., Dang, Y., Bhat, S., Merrick, W.C., Green, R., Shen, B., and Liu, J.O. (2010). Inhibition of eukaryotic translation elongation by cycloheximide and lactimidomycin. Nat Chem Biol 6, 209-217. 10.1038/nchembio.304.

Smith, B.E., Wang, S.L., Jaime-Figueroa, S., Harbin, A., Wang, J., Hamman, B.D., and Crews, C.M. (2019). Differential PROTAC substrate specificity dictated by orientation of recruited E3 ligase. Nat Commun 10, 131. 10.1038/s41467-018-08027-7. 
Sun, X., Gao, H., Yang, Y., He, M., Wu, Y., Song, Y., Tong, Y., and Rao, Y. (2019). PROTACs: great opportunities for academia and industry. Signal Transduct Target Ther 4, 64. 10.1038/s41392-019-01016.

Wagner, K.M., McReynolds, C.B., Schmidt, W.K., and Hammock, B.D. (2017). Soluble epoxide hydrolase as a therapeutic target for pain, inflammatory and neurodegenerative diseases. Pharmacol Ther 180, 62-76. 10.1016/j.pharmthera.2017.06.006.

Wang, W., Yang, J., Zhang, J., Wang, Y., Hwang, S.H., Qi, W., Wan, D., Kim, D., Sun, J., Sanidad, K.Z., et al. (2018). Lipidomic profiling reveals soluble epoxide hydrolase as a therapeutic target of obesityinduced colonic inflammation. Proc Natl Acad Sci U S A 115, 5283-5288. 10.1073/pnas.1721711115. Wang, Y., Wagner, K.M., Morisseau, C., and Hammock, B.D. (2021). Inhibition of the Soluble Epoxide Hydrolase as an Analgesic Strategy: A Review of Preclinical Evidence. J Pain Res 14, 61-72. 10.2147/JPR.S241893.

Zou, Y., Ma, D., and Wang, Y. (2019). The PROTAC technology in drug development. Cell Biochem Funct 37, 21-30. 10.1002/cbf.3369. 
Table 1. Structure of sEH-PROTAC molecules and their inhibitory potency against sEH

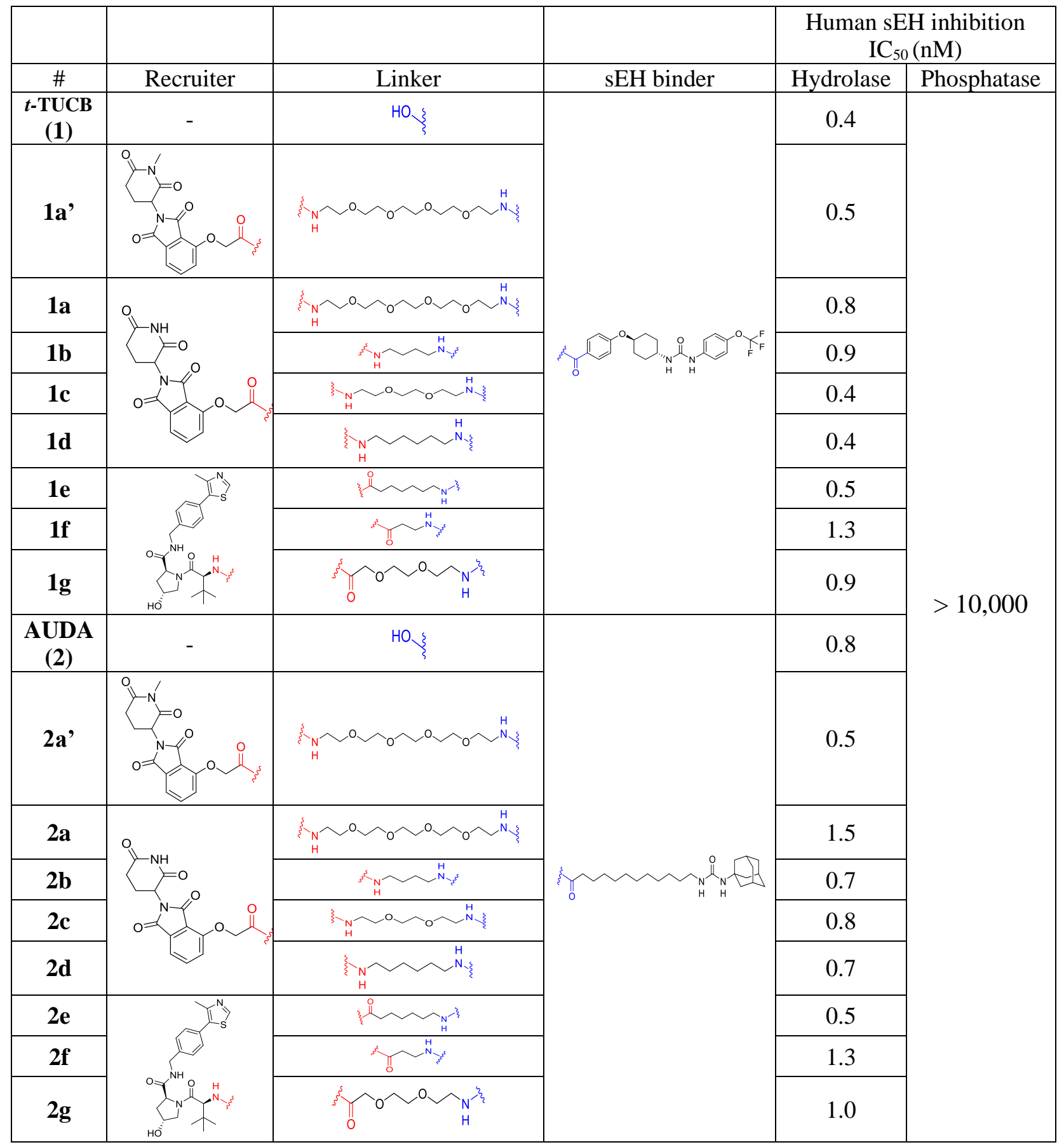




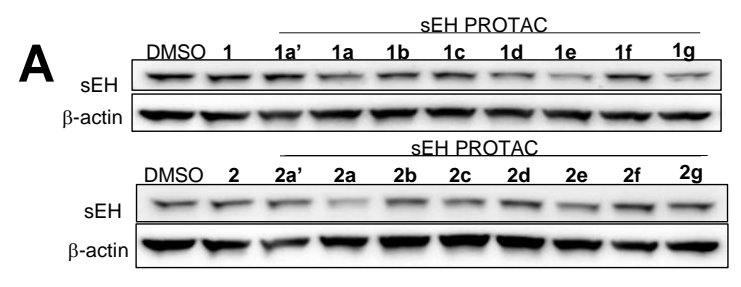

B
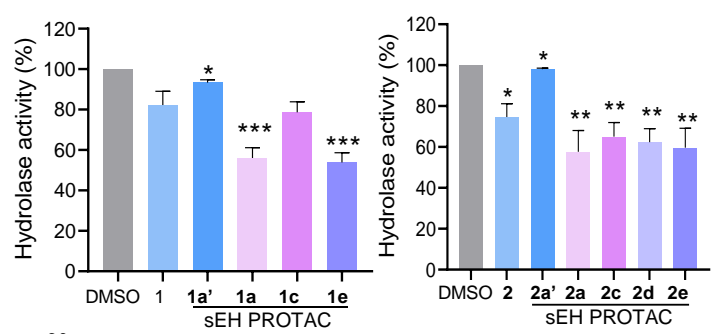

C
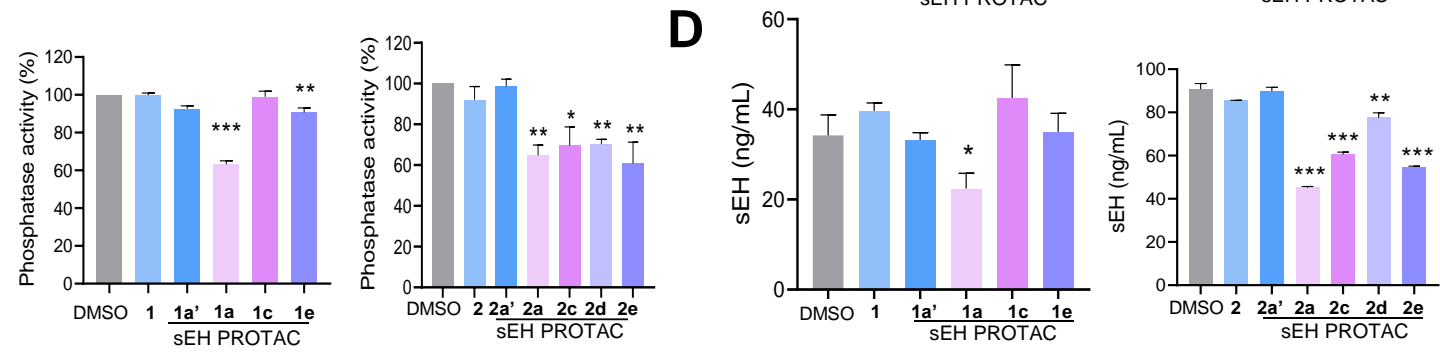

$\mathbf{E}$

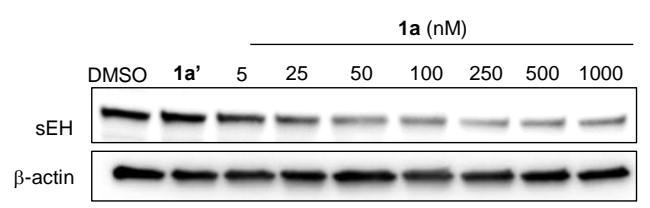

$\mathbf{F}$
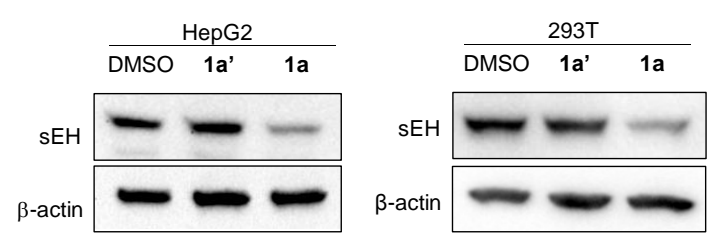

Figure 1. Effect of PROTAC compounds on sEH levels. $\mathrm{HepG}_{2}$ cells were treated with indicated compound at $1 \mu \mathrm{M}$ for 24 hours, and the sEH level was measured by (A) immunoblot (B) hydrolase activity (C) phosphatase activity (D) ELISA. The structures of sEH PROTAC compounds are shown in Table 1. Mean \pm SEM are shown. The difference between treatment groups were analyzed by one-way ANOVA with Holm Sidak test, and the normality was tested by ShapiroWilk test. If there is no significant difference using one-way ANOVA, Student's t-test was performed. The $*$ in figures $1 \mathrm{~B}, 1 \mathrm{C}$, and $1 \mathrm{D}$ represents the significant difference between the compound treatment vs DMSO treatment $(*: P<0.05, * *: P<0.01, * * *: P<0.001)$. All of the $P$ values and other statistical details were shown in Table S2. (E) The concentration response of 1a. $\mathrm{HepG}_{2}$ cells were treated with 1a for 24 hours and sEH level was measured by immunoblot. (F) Compound 1a is effective in both $\mathrm{HepG}_{2}$ and $293 \mathrm{~T}$ cells. $\mathrm{HepG}_{2}$ or $293 \mathrm{~T}$ cells were treated with DMSO, $250 \mathrm{nM} 1 \mathbf{a}$ ', or 1a for 24 hours, and sEH level was measured by immunoblot. 
A

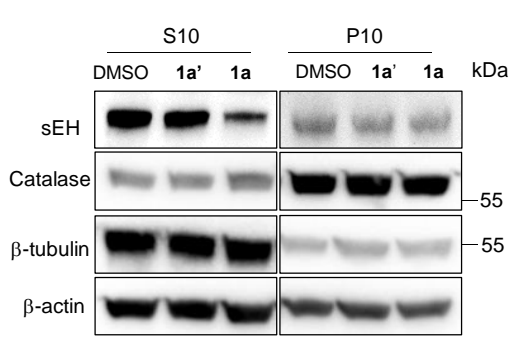

C

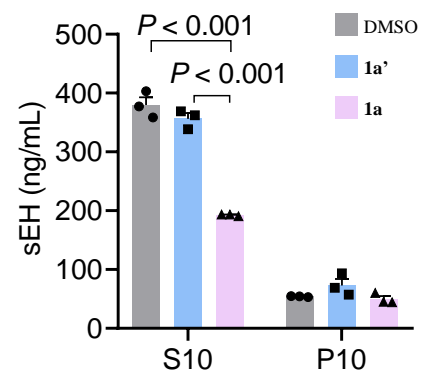

B

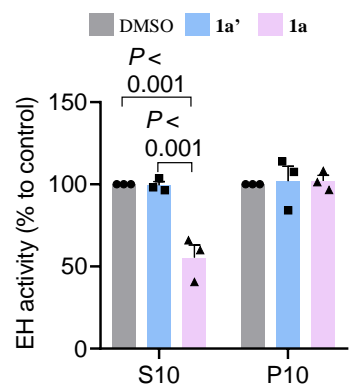

D

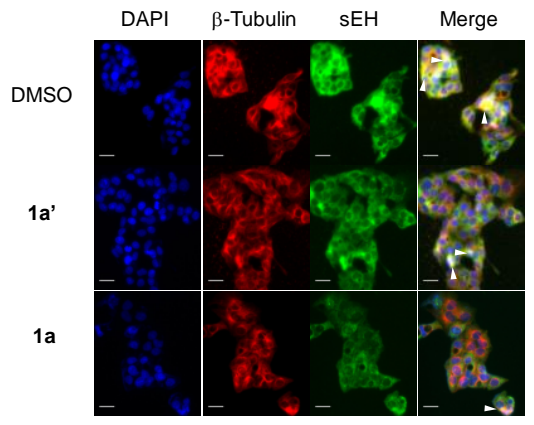

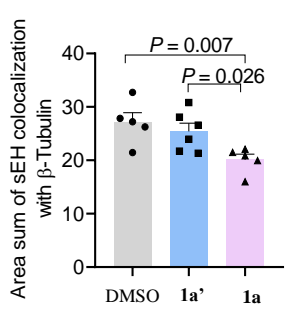

Figure 2. Compound 1a selectively degrades cytosolic sEH but not peroxisomal sEH. $\mathrm{HepG}_{2}$ cells were treated with indicated compound at $250 \mathrm{nM}$ for 24 hours and sEH levels in cytosol and peroxisome fractions were measured by $(\mathbf{A})$ immunoblot $(\mathbf{B})$ epoxide hydrolase activity $(\mathbf{C})$ ELISA. (D) Immunofluorescence results of colocalization of sEH with $\beta$-Tubulin. Scale bar, 25 $\mu \mathrm{m}$. Left panel, the representative pictures of immunofluorescence; Right panel, the quantification of immunofluorescence results. Mean \pm SEM are shown. The difference between DMSO, 1a', and 1a was analyzed using one-way ANOVA. 


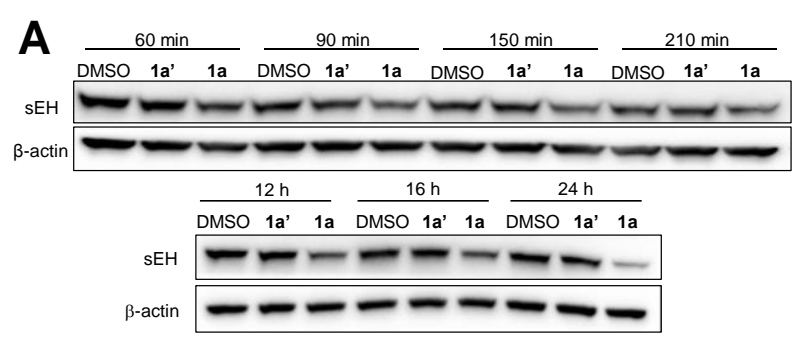

B

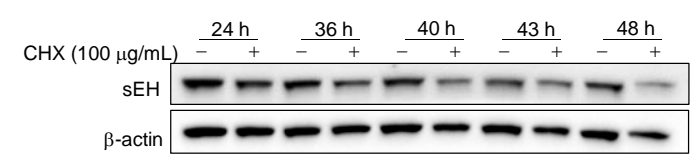

C
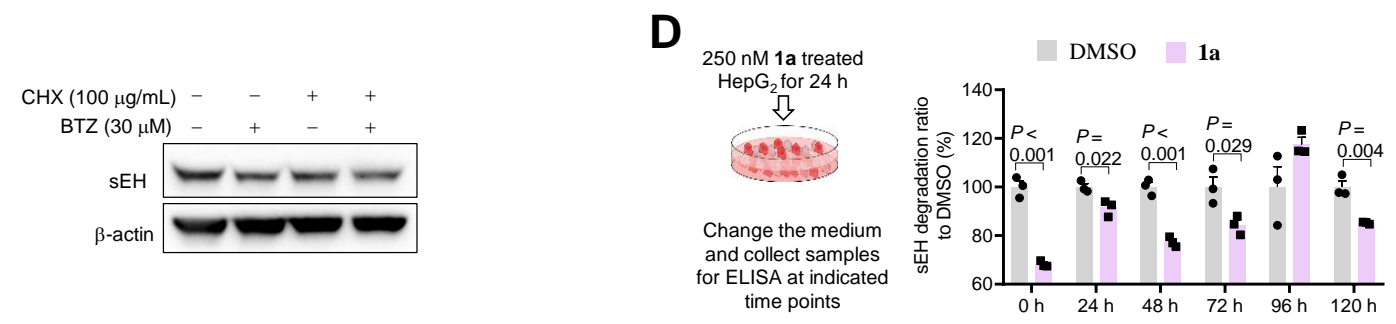

Figure 3. Degradation and production kinetics of sEH. (A) Degradation kinetics of sEH induced by PROTAC 1a. $\mathrm{HepG}_{2}$ cells were treated with indicated compound at $250 \mathrm{nM}$ and sEH level was determined by immunoblot. (B) Endogenous degradation kinetics of sEH. $\mathrm{HepG}_{2}$ cells were treated with protein synthesis inhibitor $\mathrm{CHX}(100 \mu \mathrm{g} / \mathrm{mL})$ and $\mathrm{sEH}$ level was measured using immunoblot at indicated time points. (C) Endogenous degradation of sEH is not inhibited by the proteasome inhibitor BTZ. $\mathrm{HepG}_{2}$ cells were treated with $\mathrm{CHX}$ with/without BTZ for 24 hours and sEH was detected using immunoblot at $24 \mathrm{~h}$. (D) Probing endogenous sEH production kinetics using PROTAC 1a. $\mathrm{HepG}_{2}$ cells were treated with $\mathbf{1 a}$ at $250 \mathrm{nM}$ for 24 hours, then 1a was removed from the media, and the $\mathrm{sEH}$ protein level was measured using immunoblot after incubating for indicated time. Left panel: the scheme of experiment; Right panel: The quantification results of 1a degradation effect. Mean \pm SEM are shown. Student's t-test or Wilcoxon-Mann-Whitney test was performed depending on the normality. 
A

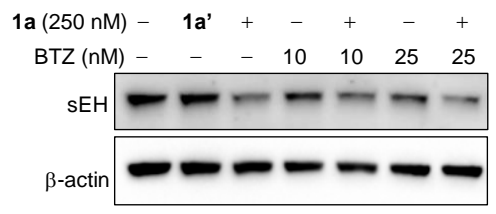

C

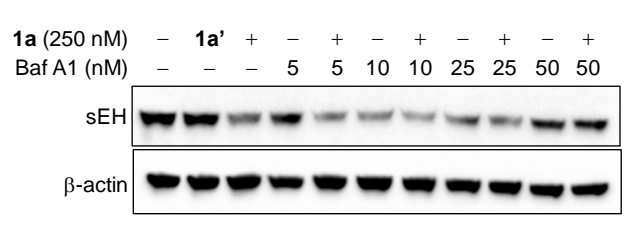

B

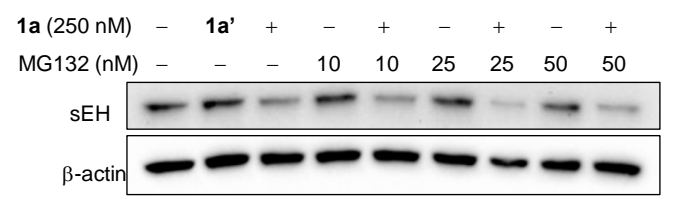

D

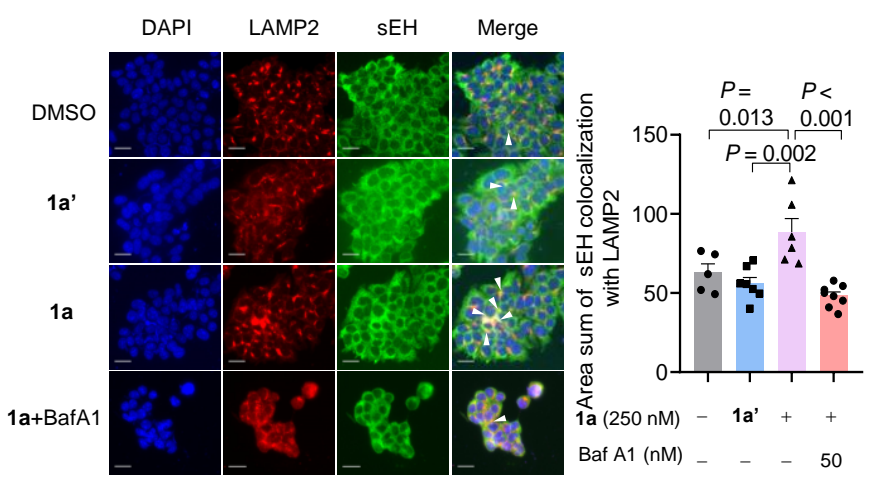

Figure 4. Compound 1a degrades SEH through a lysosome dependent pathway. The immunoblot results of sEH levels in $\mathrm{HepG}_{2}$ cells treated for 24 hours with $\mathbf{1 a}(250 \mathrm{nM})$ together with: (A) proteasome inhibitor BTZ (B) proteasome inhibitor MG132 (C) lysosome inhibitor BafA1. (D) Immunofluorescence results of the colocalization of sEH with LAMP2. Left panel: the representative images of immunofluorescence; Right panel: the quantification results of immunofluorescence. The area of colocalization was normalized to the number of cells in the corresponding images. Scale bar, $25 \mu \mathrm{m}$. Mean \pm SEM are shown. The difference between DMSO, 1a', and 1a were analyzed using one-way ANOVA. The difference between 1a, and 1a with BafA1 was analyzed by Student's t-test. 


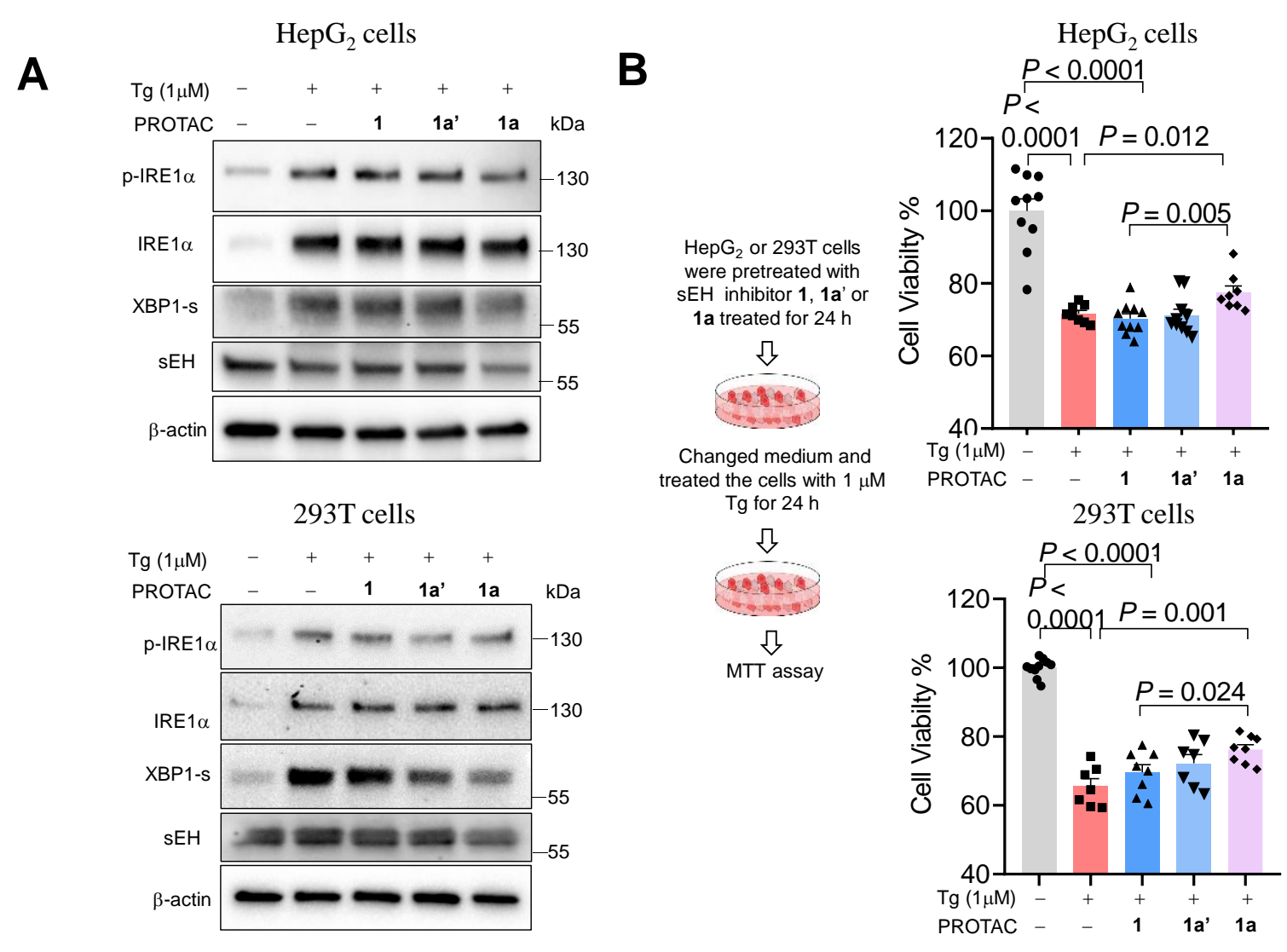

Figure 5. Compound 1a reduces ER stress through inhibition of IRE1 $\alpha$-XBP1 signaling pathway. (A) The $\mathrm{HepG}_{2}$ or $293 \mathrm{~T}$ cells were treated with $\mathrm{Tg}$, sEH inhibitor 1, 1a', or 1a for 24 hours, the immunoblot results of the proteins in IRE1 $\alpha$-XBP1 pathway. (B) The $\mathrm{HepG}_{2}$ or 293T cells were pre-treated with sEH inhibitor 1, 1a', or 1a $(250 \mathrm{nM})$ for 24 hours, then changed the medium containing Tg without PROTACs and incubated the cells for another 24 hours. The cell death was measured using MTT assay. Left panel, the procedure scheme of the treatment; Right panel, MTT assay results. $\mathrm{n}=7-10$. Tg, thapsigargin. Mean \pm SEM are shown. The difference between two groups was analyzed by Student's t-test. 